\title{
Tiludronate Disodium
}

National Cancer Institute

\section{Source}

National Cancer Institute. Tiludronate Disodium. NCI Thesaurus. Code C61973.

The disodium salt form of tiludronate, a first-generation, non-nitrogenous bisphosphonate analogue of endogenous pyrophosphate. Tiludronate adsorbs to hydroxyapatite cells, and although the exact mechanism through which this bisphosphonate exerts its effect has yet to be fully elucidated, tiludronate appears to inhibit protein-tyrosine-phosphatase (PTP) in osteoclasts, thereby disrupting the cytoskeletal ring structure and suppressing the bone resorbing activity of osteoclasts. In addition, tiludronate appears to inhibit the vacuolar-type proton ATPase (V-ATPase) in osteoclasts. Altogether, this may reduce the number of osteoclasts, inhibit abnormal bone resorption and reduce bone turnover. 